\title{
Jet cross sections and transverse momentum distributions with NNLOJET
}

\section{T. Gehrmann*, X. Chen}

Department of Physics, University of Zürich, CH-8057 Zürich, Switzerland

\section{J. Cruz-Martinez, J.R. Currie, E.W.N. Glover, T.A. Morgan, J. Niehues, D.M. Walker} Institute for Particle Physics Phenomenology, Department of Physics, University of Durham, Durham, DH1 3LE, UK

\section{R. Gauld, A. Gehrmann-De Ridder}

Institute for Theoretical Physics, ETH, CH-8093 Zürich, Switzerland

\section{A. Huss}

CERN Theory Division, CH-1211 Geneve 23, Switzerland

\section{J. Pires}

Centro de Fisica Teorica de PartŠculas - CFTP, Instituto Superior Tecnico IST, Universidade de Lisboa, Av. Rovisco Pais, P-1049-001 Lisboa, Portugal

\begin{abstract}
This talk discusses recent results for next-to-next-to-leading order (NNLO) QCD corrections to jet cross sections and transverse momentum distributions. The results are obtained in the NNLOJET code framework, which provides an implementation of the antenna subtraction method for the handling of infrared singular contributions at NNLO. We briefly describe the NNLOJET implementation, with particular emphasis on the construction of the real radiation phase space, which is tailored to ensure stability in all infrared sensitive regions.
\end{abstract}

13th International Symposium on Radiative Corrections 24-29 September, 2017

St. Gilgen, Austria

\footnotetext{
* Speaker.
} 


\section{Introduction}

Low multiplicity final states containing jets and/or gauge bosons are produced copiously at hadron colliders. Production cross sections and kinematical distributions can be measured to high accuracy, thereby enabling precision studies of Standard Model parameters. These studies rely on a detailed comparison of the experimental measurements with theory predictions, that are therefore required to match the accuracy of the experimental data. For many benchmark observables, this implies going beyond the common standard of next-to-leading order (NLO) in QCD and the electroweak theory. Important progress has been made in the past years in the calculation of nextto-next-to-leading order (NNLO) QCD corrections to collider observables [1-23]. In this talk, we report some results obtained using the NNLOJET framework, which is an implementation of the antenna subtraction method $[24,25]$ for NNLO QCD calculations.

\section{The NNLOJET framework}

At NNLO in QCD, collider observables receive three generic types of corrections to the basic Born-level process: double real (RR) corrections, real-virtual (RV) corrections and double virtual (VV) corrections. They are individually infrared divergent, and only their sum becomes finite. To obtain predictions that are fully differential in the final state kinematics, and that take proper account of the experimental jet reconstruction algorithm, the three contributions need to be processed separately. Several techniques for the extraction and recombination of infrared divergent contributions have been derived and implemented in actual calculations [24-30]. NNLOJET uses the antenna subtraction method, which constructs infrared subtraction terms for the RR, RV and VV contributions from so-called antenna functions [24, 25, 31-33] that describe the unresolved parton radiation off a pair of hard radiator partons.

The NNLOJET code is a parton-level event generator that provides the framework for the implementation of jet production processes to NNLO accuracy, using the antenna subtraction method. Besides containing the event generator infrastructure (Monte Carlo phase-space integration, event handling and analysis routines), it supplies the unintegrated and integrated antenna functions and the phase-space mappings relevant to all kinematical situations. The implementation of processes in the NNLOJET framework requires the availability of the matrix elements for all RR, RV, and $\mathrm{VV}$ processes, and the construction of the antenna subtraction terms. NNLOJET provides testing routines to verify the point-wise convergence of the subtraction, as documented for example in Ref. [34]. Processes included in NNLOJET up to now are $Z$ and $Z+j$ production [10-13], $W$ and $W+j$ production [9], $H$ and $H+j$ production [7], di-jet production in hadron-hadron collisions $[20,21]$ and in lepton-hadron collisions [22,23], as well as three-jet production in electronposition annihilation [35]. We discuss several recent phenomenological applications in Section 4 below.

NNLOJET supports parallel computing infrastructures through the OpenMP interface, which is especially crucial for the Monte Carlo warmup (adaptation of integration grids). Typical runtimes for NNLO predictions of kinematical distributions in $2 \rightarrow 2$ processes range between $80^{\prime} 000$ and 250 ' 000 core-hours, with most of the computation time spent on the double real radiation processes. 


\section{Phase space generation at NNLO}

In using the antenna subtraction method to construct subtraction terms for higher order calculations, one encounters the problem of angular correlations in the collinear splitting of a gluon into massless partons. These angular correlations introduce non-factorizing terms which correlate the hard reduced matrix element with the splitting functions. They vanish when the azimuthal variable of the collinear system (with respect to the collinear axis, defined by the collinear momentum and a light-like recoil momentum) is integrated out. The same cancellation can also be accomplished [36] by combining two collinear configurations related by an azimuthal rotation of $\pi / 2$. To specify the frame in which the rotation is performed, two light-like directions must be specified: one is given by the collinear momentum of the pair, while the other can be chosen arbitrarily.

The NNLOJET phase space generators for different processes produce pairs of phase space points related by angular rotation around a specified axis. In the following, we describe the phase space implementation using vector-boson-plus-jet production as an example process. We consider the basic kinematical situation

$$
p_{a}+p_{b} \rightarrow p_{1}+p_{2}+p_{3}\left(+p_{4}\right)\left(+p_{5}\right)
$$

where the outgoing momenta $p_{1}$ and $p_{2}$ represent the decay leptons that are not involved in any unresolved limit.

To denote the angular rotations, we introduce a shorthand notation. At NLO, we can have one collinear splitting, and thus one collinear pair to be rotated. The notation

$(i, j ; k)$ represents the rotation of the momentum pair $p_{i}, p_{j}$ around the axis defined by $p_{k}$. The corresponding phase space contains $s_{i j}$ as one of its basic variables.

The result is two complete sets of final state momenta: unrotated and rotated.

At NNLO, two pairs of partons can become collinear, or three partons can become simultaneously collinear. We distinguish two cases:

$(i ; j, k ; l)$ is the rotation appropriate to triple collinear splitting. The momenta $p_{j}$ and $p_{k}$ are first rotated around the $p_{l}$ axis, and the resulting system of $p_{i}, p_{j}, p_{k}$ is then also rotated around the $p_{l}$ axis. The phase space is constructed from a sequential splitting $(i j k) \rightarrow i+(j k) \rightarrow i+j+k$ with $s_{i j k}$ and $s_{j k}$ as the basic phase space variables.

$(i, k ; l, m ; n)$ is the rotation appropriate to double single-collinear splitting. The momenta $p_{i}$, $\left(p_{l}+p_{m}\right)$ are rotated around the $p_{k}$ axis, while the momenta $p_{l}, p_{m}$ are rotated around the $p_{n}$ axis. The phase space is again constructed from a sequential splitting $(i l m) \rightarrow i+(l m) \rightarrow i+l+m$.

In both cases, the output consists of four complete sets of final state momenta: the original momenta, only the first pair rotated, only the second pair rotated, and both pairs rotated.

The implemented phase space parametrizations are thus optimized to account for the angular rotations in a specific single or double unresolved limit. They are not appropriate for the fully inclusive coverage of the phase space. Instead, we introduce phase space wedges, which are specified 
through a set of selection criteria, such that the full final state phase space of a given multiplicity can be obtained by summing over all possible wedges. Each wedge only contains those unresolved limits to which the angular rotation is appropriate. The weight from phase space generator is the same for the original and rotated momentum set, while the matrix element and subtraction terms are evaluated separately for both sets. The final weight is obtained by summing over all events (wedges and their rotations), and dividing by the number ( 2 or 4 ) of angular rotated partner events.

The wedges are defined by imposing constraints on the invariants, which for this purpose are taken to be the $s_{i j}=2 p_{i} \cdot p_{j}$ with $\left\{p_{i}, p_{j}\right\} \in\left\{p_{a}, p_{b}, p_{3}, p_{4}, p_{5}\right\}$. In the list of all $s_{i j}$, we denote the two smallest invariants by $s_{1}$ and $s_{2}$.

\subsection{Three-particle phase space}

The three-particle final state is free of singularities, as ensured by the final state selection cuts, requiring the vector boson and a jet at finite transverse momentum.

\subsection{Four-particle phase space}

The four-particle contribution to vector boson-plus-jet production contains simple collinear initial or final state radiation. To properly account for the corresponding angular terms in some of the limits, we consider the rotation $(3,4 ; a)$. This rotation takes proper account of the angular terms in the collinear limits $(3 \| 4),(3 \| a)$ and $(4 \| a)$. The phase space routine therefore fails to take proper account of the angular terms in the initial state collinear limits involving $p_{b}$. Therefore, we restrict it to the following phase space wedge:

$$
s_{1}=s_{a 3} \text { or } s_{1}=s_{a 4}
$$

which singles out the three above-mentioned collinear limits, and can therefore also be identified by the notation $(3,4 ; a)$. The full phase space is then obtained by summing over two wedges: $(3,4 ; a),(3,4 ; b)$, the angular average is obtained by averaging over the angular partner events in each wedge.

\subsection{Five-particle phase space}

The five-particle contribution to vector-boson-plus-jet production can contain triple-collinear and double single-collinear configurations. We decompose the full five-particle phase space into 6 triple-collinear and 6 double single-collinear wedges. The full phase space is recovered by summing over all wedges. The phase space integration is structured such that two separate integrals (1. sum of triple-collinear wedges and 2. sum of double single-collinear wedges) must be evaluated to obtain the full five-particle phase space.

For the angular terms in triple-collinear configurations, we consider the rotation $(3 ; 4,5 ; a)$ This rotation takes proper account of the angular terms in the limits (3 || 4|| 5$),(a\|4\| 5)$ and (4 || 5, 3 soft). To ensure that only these limits are covered by the phase space generator, its application is restricted to a particular wedge, which is defined by

$$
\begin{aligned}
& \left(\left\{s_{1}, s_{2}\right\} \in\left\{s_{45}, s_{a 4}, s_{a 5}\right\}\right) \text { or } \\
& \left(s_{1}=s_{45} \text { and } s_{2}=\min \left(s_{34}, s_{35}\right) \text { and } s_{a 4}+s_{a 5}<s_{b 4}+s_{b 5}\right) .
\end{aligned}
$$


All six triple-collinear wedges are then obtained from $(i ; j, k ; a)$ by permuting $i$ over the three partonic final state momenta, and interchanging $(a, b)$. Full phase space coverage for the triplecollinear wedges is obtained by summing the six wedges, and the angular average is obtained by averaging with the angular partner events.

To properly account for the angular terms in double single-collinear configurations, we consider the rotation $(3 ; a ; 4,5 ; b)$. This rotation takes proper account of the angular terms in $(3\|a ; 4\|$ $5)$, ((4 or 5$)\|b ; 3\| a)$. To ensure that only these limits are covered by the phase space generator, its application is restricted to a particular wedge, which is defined by

$$
\begin{aligned}
& \left(s_{1}=s_{a 3} \text { and } s_{2}=\min \left(s_{b 3}, s_{b 4}, s_{b 5}\right)\right. \text { or } \\
& \left(\left\{s_{1}, s_{2}\right\} \in\left\{s_{45}, s_{a 3}\right\}\right) .
\end{aligned}
$$

All 6 double single-collinear wedges are then obtained from $(i ; a ; k, l ; b)$ by permuting $i$ over the three partonic final state momenta and interchanging $(a, b)$. Full phase space coverage for the double single-collinear wedges is obtained by summing the six wedges, the angular average is obtained by averaging with the angular partner events.

The full phase space is obtained by summing the integration results from the sum of the triplecollinear wedges and the sum of the double single-collinear wedges.

\section{Recent applications}

\subsection{Transverse momentum distributions in $Z$ boson production}

The production of Z-bosons which subsequently decay into a pair of leptons is a Standard Model benchmark process at hadron colliders. It occurs with a large rate and, due to its clean final state signature, can be measured very accurately with small experimental uncertainties. It has been studied extensively at the LHC by the ATLAS [37,38], CMS [39, 40] experiments. A key observable in these measurements is the transverse momentum distribution of the Z-boson, which is very sensitive to the gluon distribution in the proton. The transverse momentum of the Z-boson is due to the emission of QCD radiation from the initial state partons. As a consequence, fixed order predictions at $\mathscr{O}\left(\alpha_{\mathrm{s}}^{2}\right)$ in perturbative QCD, which are NNLO-accurate for the inclusive Z-boson production cross section correspond only to NLO-accurate predictions for the transverse momentum distributions. The perturbative description of the transverse momentum distribution of the Z-boson is therefore most closely related to $\mathrm{Z}+$ jet production, with the jet reconstruction replaced by a transverse momentum cut on the Z-boson.

Using NNLOJET, we computed the transverse momentum distributions for Z-boson production [11,12], and most recently also for $\mathrm{W}$-boson production [9]. In the small $p_{\mathrm{T}}^{\mathrm{Z}}$ region, the precision of direct measurements of the $p_{\mathrm{T}}^{\mathrm{Z}}$ spectrum using the standard $p_{\mathrm{T}}^{\mathrm{Z}}$ variable is limited by the experimental resolution on $p_{\mathrm{T}}^{\mathrm{Z}}$ itself, and in particular on the resolution of the magnitude of the transverse momenta of the individual leptons entering $p_{\mathrm{T}}^{\mathrm{Z}}$. To probe the low $p_{\mathrm{T}}^{\mathrm{Z}}$ domain of $\mathrm{Z} / \gamma^{*}$ production an alternative angular variable, $\phi_{\eta}^{*}$, has been proposed [41]. This variable is reconstructed entirely from the lepton directions (without using the lepton energies) and therefore minimises the impact of these experimental uncertainties. At low $p_{\mathrm{T}}^{\mathrm{Z}}$, one finds the approximate relation:

$$
2 \phi_{\eta}^{*} \approx p_{\mathrm{T}}^{\mathrm{Z}} / m_{\ell \ell} .
$$



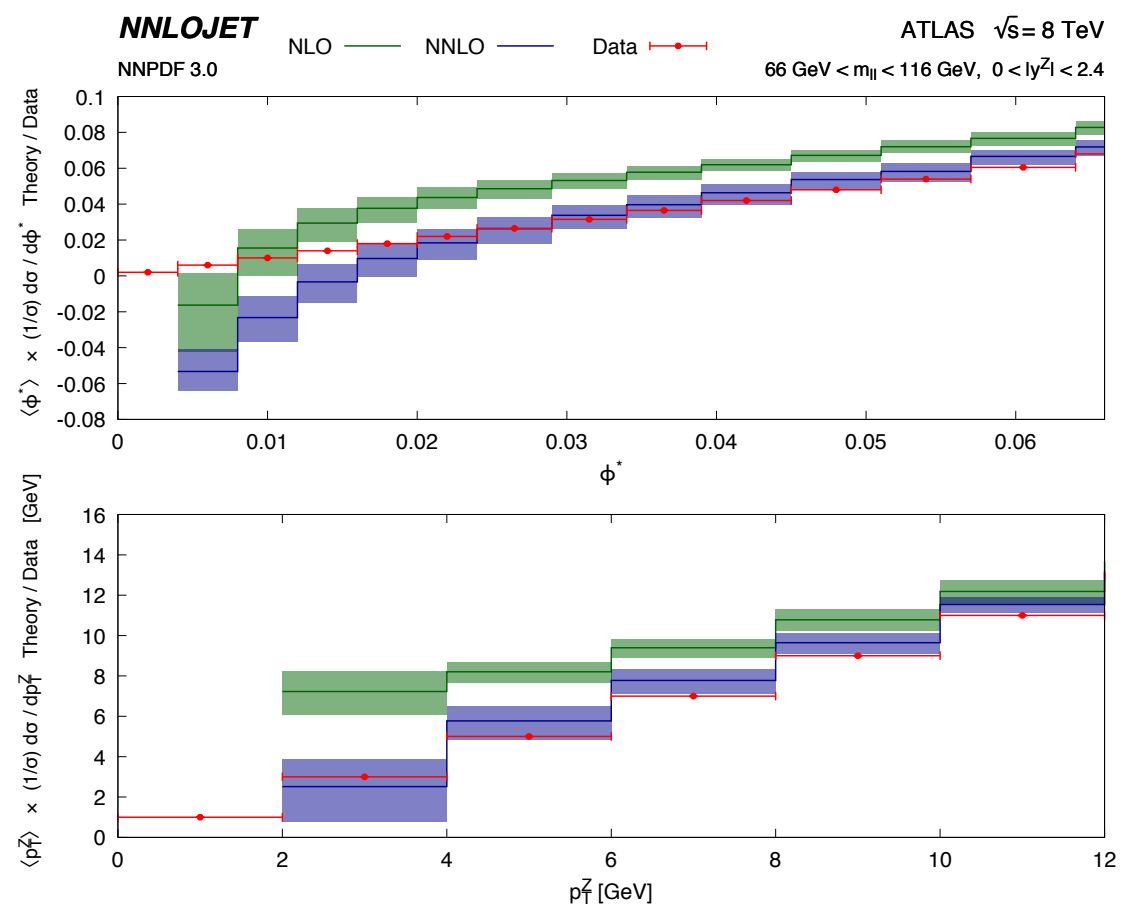

Figure 1: The $\phi_{\eta}^{*}$ and $p_{\mathrm{T}}^{\mathrm{Z}}$ distributions for the on-resonance mass bin $66 \mathrm{GeV}<m_{\ell \ell}<116 \mathrm{GeV}$. The distribution is normalised to the experimental ATLAS data [38]. The green bands denote the NLO prediction with scale uncertainty and the blue bands show the NNLO prediction with scale uncertainty. Figure from [12].

The distributions in $\phi_{\eta}^{*}$ and $p_{\mathrm{T}}^{\mathrm{Z}}$ are therefore closely related in the infrared region. In particular, one should expect the onset of large logarithmic corrections (and consequently the breakdown of the fixed order NLO and NNLO predictions) to occur roughly simultaneously at values of $\phi_{\eta}^{*}$ and $p_{\mathrm{T}}^{\mathrm{Z}}$ related through the above relation. To illustrate this, Fig. 1 superimposes the infrared regions of these distributions. The $p_{T}^{Z}$ range is fixed to $[0,12] \mathrm{GeV}$, while the $\phi_{\eta}^{*}$ range is chosen according to Eq. (4.1). The first bins contain the zero value and are not accessible by a fixed-order calculation of the $p_{\mathrm{T}}^{\mathrm{Z}}$ or $\phi_{\eta}^{*}$ distributions, which diverges there.

First and foremost, we observe the substantially higher experimental resolution in $\phi_{\eta}^{*}$. This reflects the much better experimental resolution of the low $p_{\mathrm{T}}^{\mathrm{Z}}$ region afforded by the $\phi_{\eta}^{*}$ variable. We see that the NLO prediction does not describe the data in the plotting range and only starts to describe the data at larger values of $p_{\mathrm{T}}^{\mathrm{Z}}$ and $\phi_{\eta}^{*}$. However, we observe that the NNLO description remains reliable down to values of $\phi_{\eta}^{*} \approx 0.02$. The precise point of deviation in the $p_{\mathrm{T}}^{Z}$ distributions cannot be resolved due to the coarse binning. Nevertheless, these values of $\phi_{\eta}^{*}$ and $p_{\mathrm{T}}^{\mathrm{Z}}$ where the fixed order predictions start to deviate from the data appear to be in line with the expectation from Eq. (4.1). A description of the full distributions in $\phi_{\eta}^{*}$ and $p_{T}^{Z}$ will require the matching of the fixed order NNLO predictions onto resummation.

Even more detailed information on the production dynamics of Z-bosons can be extracted from the angular coefficients that determine the full kinematical distribution of the decay leptons. Also for these angular coefficients, we observe that inclusion of NNLO QCD corrections results in a 
stabilization of the theoretical predictions and a considerably better description of the experimental data [13]. Together with the transverse momentum distributions of $\mathrm{Z}$ and $\mathrm{W}$ bosons, these angular coefficients play an outstanding role in the determination of the W-boson mass from lepton-plusmissing energy distributions. With our NNLOJET implementations [9,11-13], predictions for all these distributions can now be obtained to NNLO accuracy.

\subsection{Jet production in deep inelastic scattering}

Our understanding of the inner structure of the proton has been shaped through a long series of deep-inelastic lepton-nucleon scattering (DIS) experiments, which have established the partonic structure of the proton and provided precision measurements of parton distribution functions (PDFs). While the quark distributions can be probed directly in inclusive DIS, gluon-initiated processes enter only as higher order corrections. A direct determination of the gluon distribution requires the selection of specific hadronic final states such as heavy quarks or jets.

The DESY HERA electron-proton collider provided a large data set of hadronic final states in DIS at $\sqrt{s}=319 \mathrm{GeV}$. Jet final states have been measured to high precision over a large kinematical range by the H1 $[42,43]$ and ZEUS [44] experiments. The reconstruction of jets is performed in the Breit frame, defined by the direction of the virtual photon and incoming proton. Jet production in the Breit frame is induced both by quarks and gluons in the initial state, with gluons making up the dominant contribution almost throughout the entire kinematical range. Up to very recently, the theoretical description of jet production in DIS was limited to NLO in QCD. At this order, the residual theory uncertainty is typically larger than the experimental errors on the HERA data, thereby limiting the impact of the data in precision QCD studies.
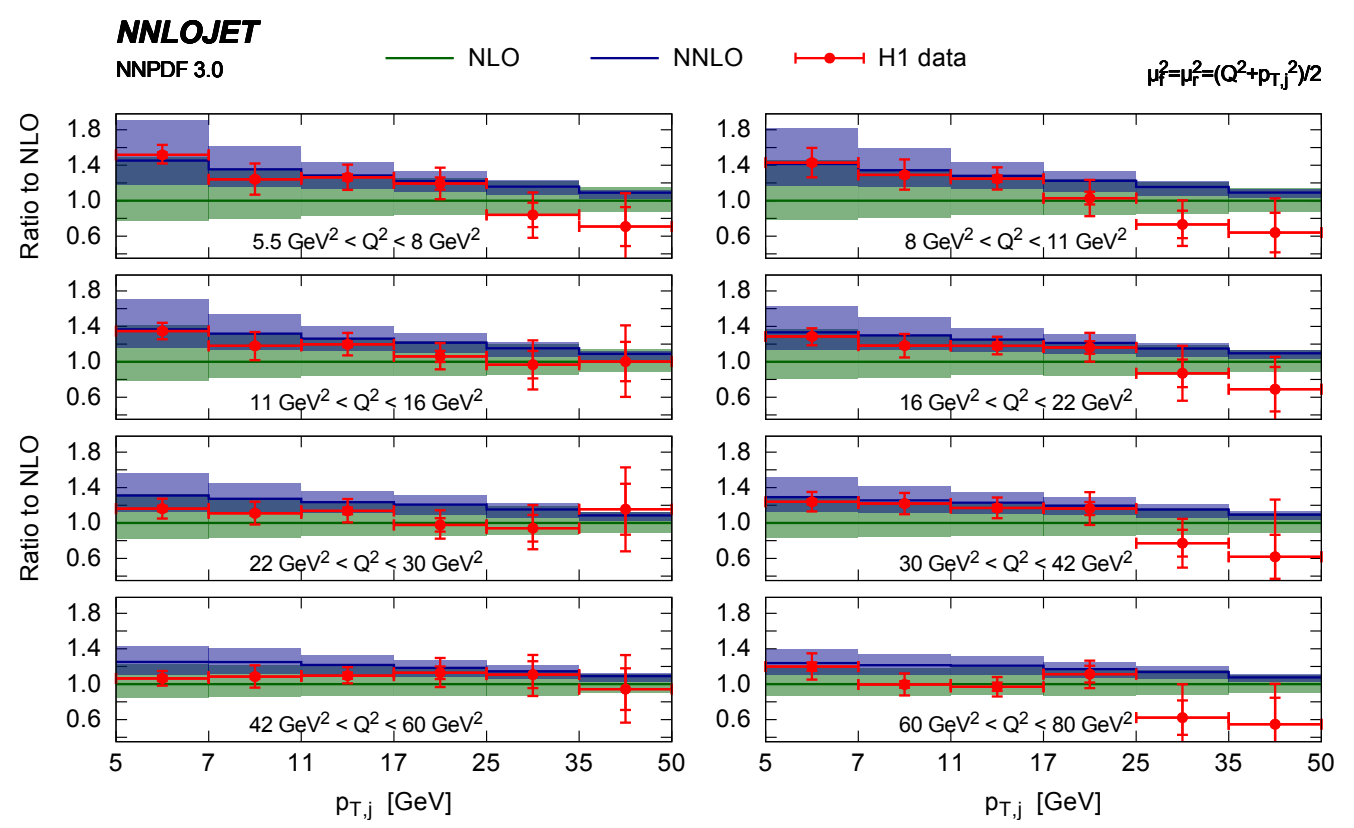

Figure 2: Inclusive jet production cross section as a function of the jet transverse momentum $p_{T, B}$ in bins of $Q^{2}$, compared to H1 data [43]. Figure from [23]. 
Using the NNLOJET framework, we computed the NNLO corrections to jet production in DIS, both for di-jet final states [22] and for single inclusive jet production [23]. Figure 2 compares our NNLO predictions to the H1 single jet inclusive data [43]. We observe that the NNLO corrections are very substantial at low- $Q^{2}$ and low- $p_{T}^{B}$, with an up to $60 \%$ enhancement with respect to NLO. These large corrections are within the NLO uncertainty band (close to the upper edge), and lead to in a residual theory uncertainty of $20 \%$ even at NNLO. Especially at low $Q^{2}$, the shape and normalisation of the theory prediction changes significantly going from NLO to NNLO, and results in a considerably improved theoretical description of the data, as already statistically quantified in the experimental H1 study [43].

Our newly derived NNLO predictions were used for a re-analysis of all $\mathrm{H} 1$ jet and di-jet data in view of an improved determination of the strong coupling constant and the gluon distribution [45]. This study is the first application of an NNLOJET interface to the ApplFast-NNLO framework that recasts the NNLO predictions in terms of parton-level coefficient functions, thereby enabling fast multiple evaluations of the predictions for varying coupling constants, scales and parton distribution functions.

Using parton distributions from the global NNPDF3.1 fit [46], the re-analysis of the H1 jet data yields the determination of the strong coupling constant [45]:

$$
\alpha_{s}\left(M_{Z}, \mathrm{H} 1 \text { jets, NNLO }\right)=0.1157(20)_{\exp }(29)_{\mathrm{th}} .
$$

Based on the same data set, a simultaneous determination of the strong coupling constant and the parton distribution functions was also performed [45], resulting in a slightly lower value of $\alpha_{s}$ with quark and gluon distributions rising more steeply at low $x$ than observed in the global fits.

\subsection{Jet production at LHC}

Hadron-hadron collisions generally lead to final states containing jets. When at least two jets are produced, the two jets leading in transverse momentum, $p_{T}$, constitute a dijet system. The two jets in the final state allow for a full reconstruction of parton-level kinematics, thereby providing valuable information on important Standard Model parameters such as the strong coupling, $\alpha_{s}$, and the PDFs. Di-jet production is being studied in detail by the LHC experiments $[47,48]$, both in view of searches for strongly interacting physics beyond the Standard Model and for precision measurements.

To fully exploit the wealth of available data it is important to have a reliable and accurate theoretical prediction. Up to very recently, jet production at hadron colliders was known to NLO accuracy in perturbative QCD. Although the NLO corrections give an improvement on the LO prediction, there remains significant theoretical uncertainty associated with the NLO calculation. It is well known that the choice of scales for renormalization, $\mu_{R}$, and factorization, $\mu_{F}$, has a big impact on the predictions at NLO and, for this reason, the dijet data is regularly excluded from global PDF fits.

NNLO corrections to jet production at hadron colliders were first computed using NNLOJET for single jet inclusive production [20], and most recently extended to di-jet observables [21]. We observe that the inclusion of the NNLO corrections results in a considerable reduction of the spread of the predictions obtained for different central scale choices. Fig. 3 shows the di-jet invariant mass distributions at $7 \mathrm{TeV}$ in different bins in rapidity, normalized to the NLO prediction. 


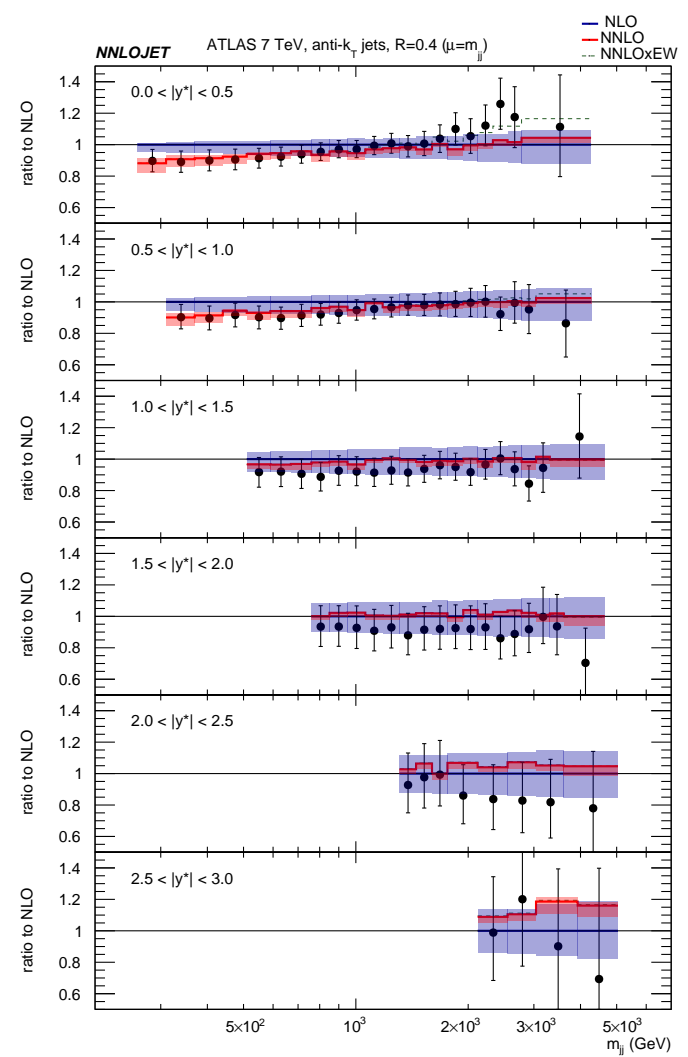

Figure 3: The NLO (blue) and NNLO (red) theory predictions and ATLAS data [48] normalized to the NLO central value. The bands represent the variation of the theoretical scales in the numerator by factors of 0.5 and 2. Electroweak effects are implemented as a multiplicative factor and shown separately as the green dashed line. Figure from [21].

Comparison with the ATLAS data [48] shows good agreement with the NNLO QCD prediction across the entire dynamical range in $m_{j j}$ and $\left|y^{*}\right|$ and a significant improvement in the description of the data for low $m_{j j}$ and $\left|y^{*}\right|$, where NLO does not adequately capture the shape nor the normalization. We include the electroweak effects as a multiplicative factor, as calculated in [49], and note that in the region where they are non-negligible $\left(\left|y^{*}\right|<0.5, m_{j j}>2 \mathrm{TeV}\right)$ they improve the description of the data. It will now be very interesting to investigate the impact of these data on a global NNLO determination of parton distributions.

\section{Conclusions and Outlook}

In this talk, we highlighted several recent results for NNLO QCD corrections to jet observables and transverse momentum distributions that were obtained using the NNLOJET framework. In general, it is observed that the NNLO predictions provide a much-improved description of the kinematical distributions observed in experimental measurements, along with a substantial reduction of the residual theoretical uncertainty.

The calculation of NNLO QCD corrections is computationally expensive. To use these results as input to an experimental analysis, new methods for their dissemination will have to be explored. 
The NNLO study of H1 deep inelastic jet data, leading to a new extraction of the strong coupling constant and a study of parton distributions, is a first example of such an application, using grid tables to parametrize the NNLO results in terms of coefficient functions.

Moreover, we note that NNLO QCD calculations are currently limited by the availability of VV matrix elements to $2 \rightarrow 2$ processes. Further progress will require a higher degree of automation in the calculation of two-loop matrix elements at higher multiplicity.

\section{Acknowledgements}

This research was supported in part by the UK Science and Technology Facilities Council, by the Swiss National Science Foundation (SNF) under contracts 200020-175595, 200021172478, and CRSII2-160814, by the Research Executive Agency (REA) of the European Union under the Grant Agreement PITN-GA-2012-316704 ("HiggsTools") and the ERC Advanced Grant MC@NNLO (340983), and by the Fundacao para a Ciencia e a Tecnologia (FCT-Portugal), project UID/FIS/00777/2013.

\section{References}

[1] S. Catani, L. Cieri, D. de Florian, G. Ferrera and M. Grazzini, Phys. Rev. Lett. 108 (2012) 072001 [arXiv:1110.2375 [hep-ph]]; J. M. Campbell, R. K. Ellis, Y. Li and C. Williams, JHEP 1607 (2016) 148 [arXiv:1603.02663 [hep-ph]].

[2] G. Ferrera, M. Grazzini and F. Tramontano, Phys. Rev. Lett. 107 (2011) 152003 [arXiv:1107.1164 [hep-ph]].

[3] M. Grazzini, S. Kallweit and D. Rathlev, JHEP 1507 (2015) 085 [arXiv:1504.01330 [hep-ph]].

[4] M. Czakon, P. Fiedler and A. Mitov, Phys. Rev. Lett. 110 (2013) 252004 [arXiv:1303.6254 [hep-ph]].

[5] M. Czakon, D. Heymes and A. Mitov, Phys. Rev. Lett. 116 (2016) 082003 [arXiv:1511.00549 [hep-ph]].

[6] R. Boughezal, F. Caola, K. Melnikov, F. Petriello and M. Schulze, Phys. Rev. Lett. 115 (2015) 082003 [arXiv:1504.07922 [hep-ph]]; F. Caola, K. Melnikov and M. Schulze, Phys. Rev. D 92 (2015) 074032 [arXiv:1508.02684 [hep-ph]].

[7] X. Chen, J. Cruz-Martinez, T. Gehrmann, E. W. N. Glover and M. Jaquier, JHEP 1610 (2016) 066 [arXiv:1607.08817 [hep-ph]].

[8] R. Boughezal, C. Focke, X. Liu and F. Petriello, Phys. Rev. Lett. 115 (2015) 062002 [arXiv:1504.02131 [hep-ph]].

[9] A. Gehrmann-De Ridder, T. Gehrmann, E. W. N. Glover, A. Huss and D. M. Walker, arXiv:1712.07543 [hep-ph].

[10] A. Gehrmann-De Ridder, T. Gehrmann, E. W. N. Glover, A. Huss and T. A. Morgan, Phys. Rev. Lett. 117 (2016) 022001 [arXiv:1507.02850 [hep-ph]].

[11] A. Gehrmann-De Ridder, T. Gehrmann, E. W. N. Glover, A. Huss and T. A. Morgan, JHEP 1607 (2016) 133 [arXiv:1605.04295 [hep-ph]].

[12] A. Gehrmann-De Ridder, T. Gehrmann, E. W. N. Glover, A. Huss and T. A. Morgan, JHEP 1611 (2016) 094 [arXiv:1610.01843 [hep-ph]]. 
[13] R. Gauld, A. Gehrmann-De Ridder, T. Gehrmann, E. W. N. Glover and A. Huss, JHEP 1711 (2017) 003 [arXiv:1708.00008 [hep-ph]].

[14] R. Boughezal, J. M. Campbell, R. K. Ellis, C. Focke, W. T. Giele, X. Liu and F. Petriello, Phys. Rev. Lett. 116 (2016) 152001 [arXiv:1512.01291 [hep-ph]].

[15] J. M. Campbell, R. K. Ellis and C. Williams, Phys. Rev. Lett. 118 (2017) 222001 [arXiv:1612.04333 [hep-ph]]; Phys. Rev. D 96 (2017) 014037 [arXiv:1703.10109 [hep-ph]].

[16] F. Cascioli et al., Phys. Lett. B 735 (2014) 311 [arXiv:1405.2219 [hep-ph]]; M. Grazzini, S. Kallweit and D. Rathlev, Phys. Lett. B 750 (2015) 407 [arXiv:1507.06257 [hep-ph]].

[17] T. Gehrmann, M. Grazzini, S. Kallweit, P. Maierhöfer, A. von Manteuffel, S. Pozzorini, D. Rathlev and L. Tancredi, Phys. Rev. Lett. 113 (2014) 212001 [arXiv:1408.5243 [hep-ph]]; M. Grazzini, S. Kallweit, S. Pozzorini, D. Rathlev and M. Wiesemann, JHEP 1608 (2016) 140 [arXiv:1605.02716 [hep-ph]].

[18] M. Grazzini, S. Kallweit, D. Rathlev and M. Wiesemann, Phys. Lett. B 761 (2016) 179 [arXiv:1604.08576 [hep-ph]].

[19] G. Abelof, R. Boughezal, X. Liu and F. Petriello, Phys. Lett. B 763 (2016) 52 [arXiv:1607.04921 [hep-ph]].

[20] J. Currie, E. W. N. Glover and J. Pires, Phys. Rev. Lett. 118 (2017) 072002 [arXiv:1611.01460 [hep-ph]];

[21] J. Currie, A. Gehrmann-De Ridder, T. Gehrmann, E. W. N. Glover, A. Huss and J. Pires, Phys. Rev. Lett. 119 (2017) 152001 [arXiv:1705.10271 [hep-ph]].

[22] J. Currie, T. Gehrmann and J. Niehues, Phys. Rev. Lett. 117 (2016) 042001 [arXiv:1606.03991 [hep-ph]].

[23] J. Currie, T. Gehrmann, A. Huss and J. Niehues, JHEP 1707 (2017) 018 [arXiv:1703.05977 [hep-ph]].

[24] A. Gehrmann-De Ridder, T. Gehrmann and E.W.N. Glover, JHEP 0509 (2005) 056 [hep-ph/0505111]; Phys. Lett. B 612 (2005) 49 [hep-ph/0502110]; Phys. Lett. B 612 (2005) 36 [hep-ph/0501291].

[25] J. Currie, E. W. N. Glover and S. Wells, JHEP 1304 (2013) 066 [arXiv:1301.4693 [hep-ph]].

[26] M. Czakon, Phys. Lett. B 693 (2010) 259 [arXiv:1005.0274]; R. Boughezal, K. Melnikov and F. Petriello, Phys. Rev. D 85 (2012) 034025 [arXiv:1111.7041 [hep-ph]].

[27] R. Boughezal, C. Focke, X. Liu and F. Petriello, Phys. Rev. Lett. 115 (2015) 062002 [arXiv:1504.02131 [hep-ph]]; R. Boughezal, X. Liu and F. Petriello, Phys. Rev. D 91 (2015) 094035; J. Gaunt, M. Stahlhofen, F. J. Tackmann and J. R. Walsh, JHEP 1509 (2015) 058 [arXiv:1505.04794 [hep-ph]].

[28] G. Somogyi and Z. Trocsanyi, JHEP 0808 (2008) 042 [arXiv:0807.0509 [hep-ph]]; V. Del Duca, C. Duhr, A. Kardos, G. Somogyi, Z. Ször, Z. Trocsanyi and Z. Tulipant, Phys. Rev. D 94 (2016) 074019 [arXiv: 1606.03453 [hep-ph]].

[29] T. Binoth and G. Heinrich, Nucl. Phys. B 693 (2004) 134 [hep-ph/0402265]; C. Anastasiou, K. Melnikov and F. Petriello, Phys. Rev. D 69 (2004) 076010 [hep-ph/0311311].

[30] S. Catani and M. Grazzini, Phys. Rev. Lett. 98 (2007) 222002 [hep-ph/0703012].

[31] A. Daleo, T. Gehrmann and D. Maitre, JHEP 0704 (2007) 016 [hep-ph/0612257]. 
[32] A. Daleo, A. Gehrmann-De Ridder, T. Gehrmann and G. Luisoni, JHEP 1001 (2010) 118 [arXiv:0912.0374 [hep-ph]].

[33] R. Boughezal, A. Gehrmann-De Ridder and M. Ritzmann, JHEP 1102 (2011) 098 [arXiv:1011.6631 [hep-ph]]; A. Gehrmann-De Ridder, T. Gehrmann and M. Ritzmann, JHEP 1210 (2012) 047 [arXiv:1207.5779 [hep-ph]]; T. Gehrmann and P. F. Monni, JHEP 1112 (2011) 049 [arXiv:1107.4037 [hep-ph]].

[34] E.W.N. Glover and J. Pires, JHEP 1006 (2010) 096 [arXiv:1003.2824 [hep-ph]]; A. Gehrmann-De Ridder, E.W.N. Glover and J. Pires, JHEP 1202 (2012) 141 [arXiv:1112.3613 [hep-ph]].

[35] T. Gehrmann, E. W. N. Glover, A. Huss, J. Niehues and H. Zhang, Phys. Lett. B 775 (2017) 185 [arXiv:1709.01097 [hep-ph]].

[36] S. Weinzierl, Phys. Rev. D 74 (2006) 014020 [hep-ph/0606008].

[37] G. Aad et al. [ATLAS Collaboration], JHEP 1409 (2014) 145 [arXiv:1406.3660 [hep-ex]].

[38] G. Aad et al. [ATLAS Collaboration], Eur. Phys. J. C 76 (2016) 291 [arXiv:1512.02192 [hep-ex]].

[39] S. Chatrchyan et al. [CMS Collaboration], Phys. Rev. D 85 (2012) 032002 [arXiv:1110.4973 [hep-ex]].

[40] V. Khachatryan et al. [CMS Collaboration], Phys. Lett. B 749 (2015) 187 [arXiv:1504.03511 [hep-ex]].

[41] A. Banfi, S. Redford, M. Vesterinen, P. Waller and T. R. Wyatt, Eur. Phys. J. C 71 (2011) 1600 [arXiv:1009.1580 [hep-ex]].

[42] V. Andreev et al. [H1 Collaboration], Eur. Phys. J. C 75 (2015) 65 [arXiv:1406.4709 [hep-ex]].

[43] V. Andreev et al. [H1 Collaboration], Eur. Phys. J. C 77 (2017) 215 [arXiv:1611.03421 [hep-ex]].

[44] S. Chekanov et al. [ZEUS Collaboration], Nucl. Phys. B 765 (2007) 1 [hep-ex/0608048].

[45] V. Andreev et al. [H1 Collaboration], Eur. Phys. J. C 77 (2017) 791 [arXiv:1709.07251 [hep-ex]].

[46] R. D. Ball et al. [NNPDF Collaboration], Eur. Phys. J. C 77 (2017) 663 [arXiv:1706.00428 [hep-ph]].

[47] S. Chatrchyan et al. [CMS Collaboration], Phys. Rev. D 87 (2013) 112002 [arXiv:1212.6660 [hep-ex]].

[48] G. Aad et al. [ATLAS Collaboration], JHEP 1405 (2014) 059 [arXiv:1312.3524 [hep-ex]].

[49] S. Dittmaier, A. Huss and C. Speckner, JHEP 1211 (2012) 095 [arXiv:1210.0438 [hep-ph]]. 